Artigo Original

\title{
Efeitos da Lipoproteína LDL-oxidada Sobre a Proliferação e a Motilidade Espontânea in Vitro de Células Endoteliais de Artérias Coronárias Humanas
}

\author{
Hermes Toros Xavier, Dulcinéia Saes Parra Abdalla, Tania Leme da Rocha Martinez, \\ José Antonio Franchini Ramires, Antonio Ricardo de Toledo Gagliardi \\ São Paulo, SP
}

\section{Objetivo}

Investigar os efeitos de baixas concentrações de LDL oxidada ( $L D L-o x)$ sobre a proliferação e a motilidade espontânea de células endoteliais de artérias coronárias humanas (CEACH) em cultura.

\section{Métodos}

Culturas de CEACH foram tratadas com baixas concentrações de $L D L$ nativa ( $L D L n)$, isolada de plasma humano, e com $L D L$ minimamente oxidada por diferentes métodos químicos, e os efeitos, comparados entre si.

\section{Resultados}

LDLn não apresentou efeitos deletérios sobre o endotélio em proliferação e na motilidade in vitro de $C E A C H$, porém na mais alta concentração e por tempo mais prolongado inibiu a proliferação celular. As LDL-ox, quimicamente, pela espermina nonoato (ENO) e 3-morfolinosidnonimina ( $S I N-1)$ expressaram efeitos inibitórios significativos sobre a proliferação e a motilidade in vitro de $\mathrm{CEACH}$ proporcionais às maiores concentrações e graus de oxidação das $L D L$.

\section{Conclusão}

LDL-ox apresenta efeito citotóxico, inibindo a proliferação e a motilidade espontânea de células endoteliais de artérias coronárias humanas em cultura, proporcionalmente à concentração e ao grau de oxidação da $L D L$, enquanto, $L D L$ nativa é relativamente inócua.

\section{Palavras-chave}

LDL-oxidada, células endoteliais de artérias coronárias humanas, doença aterosclerótica cardiovascular.

Instituto do Coração da Faculdade de Medicina da USP e Faculdade de Ciências Farmacêuticas da USP

Endereço para Correspondência: Hermes Toros Xavier

Av. Bartolomeu de Gusmão, 178/73 - Cep 11030-500 - Santos, SP E-mail: htxavier@cardiol.br

Recebido para publicação em: 11/06/2003
Níveis plasmáticos elevados de lipoproteína de baixa densidade (LDL) são considerados um dos principais fatores de risco para o desenvolvimento de doença aterosclerótica cardiovascular. A hipótese da resposta à injúria da aterosclerose propõe que o primeiro passo na aterogênese é a disfunção endotelial induzida pela ação dos fatores de risco, em especial, pela exposição do endotélio vascular à LDL oxidada (LDL-ox) ${ }^{1}$.

Estudos preliminares revelaram que preparações de LDL humana isolada exercem efeitos tóxicos sobre células endoteliais em cultura $^{2-4}$. As LDL nativas (LDLn) sofrem alterações químicas relacionadas com a peroxidação de ácidos graxos polinsaturados, constituintes da lipoproteína, que resultam em um grande aumento na sua susceptibilidade à fagocitose e à degradação por macrófagos ${ }^{5}$.

Foi demonstrado in vitro que os principais tipos de células da parede vascular, as células endoteliais, as células musculares lisas e os macrófagos são capazes de oxidar a LDL nativa ${ }^{6}$. A LDL oxidada inibi a migração das células endoteliais, mecanismo essencial nos processos de restabelecimento da integridade vascular após dano na angiogênese, de forma proporcional à concentração e ao grau de oxidação da LDL, mediado pela formação dos hidroperóxidos lipídicos ${ }^{7}$.

Estudos recentes demonstraram que os componentes lipídicos da LDL-ox ${ }^{8,9}$ causariam um aumento parodoxal na produção do fator de crescimento do endotélio vascular (VEGF) por células endoteliais em cultura, um provável mecanismo protetor frente à agressão celular. Foi observado um duplo efeito da LDL-ox, induzindo proliferação celular em baixas concentrações (5 a $10 \mu \mathrm{g} / \mathrm{mL}$ ) e apoptose em concentrações acima de $50 \mu \mathrm{g} / \mathrm{mL}^{10}$.

As células endoteliais constituem uma população heterogênea. Células endoteliais oriundas de diversos leitos vasculares expressam antígenos de superfície característicos, transportadores intracelulares específicos e regulação de enzimas intracelulares diferentes ${ }^{11}$.

Considerando a importância da heterogeneidade da célula endotelial, os dados contraditórios da literatura e visando contribuir para um melhor entendimento dos processos básicos que envolvem a interação LDL-ox e célula endotelial, realizamos o primeiro estudo sistematizado utilizando células endoteliais de artérias coronárias humanas $(\mathrm{CEACH})$ em cultura, cujo papel na aterogênese coronária é primordial.

Investigamos e descrevemos os efeitos de baixas concentrações das lipoproteínas LDLn e LDL-ox quimicamente por diferentes métodos e tempos de oxidação sobre a proliferação celular e a motilidade espontânea in vitro. 


\section{Métodos}

Utilizamos a linhagem celular de células endoteliais de artérias coronárias humanas obtidas através da CLONETICS (BioWhittaker Inc., Walkersville, MD/USA). O meio de cultura utilizado foi MCDB131 (GIBCO) adicionado de 10 ng/ml EGF (INTERGEN), 1,0 $\mu \mathrm{g} /$ $\mathrm{mL}$ hidrocortisona (SIGMA), $50 \mu \mathrm{g} / \mathrm{mL}$ de anfotericina B (GIBCO), $100 \mathrm{U} / \mathrm{mL}$ de penicilina e de estreptomicina (GIBCO) e 10\% de soro fetal bovino (SFB) (GIBCO) adicionado de L-glutamina $1 \mu \mathrm{M}$ (GIBCO). Os experimentos foram realizados em meio contendo $5 \%$ de SFB.

A contagem de células para a avaliação da proliferação e migração celular foi feita sob microscopia direta (Nikon-TMS) com aumento de 100 vezes e realizada em três campos microscópicos distintos representativos para cada placa e calculada a média de células visualizadas.

O ensaio de migração celular foi realizado de acordo com o modelo de cicatrização in vitro, estabelecido por Burk ${ }^{12}$. Em resumo, as células endoteliais foram cultivadas em placas de cultura de $60 \mathrm{~mm}$ (FALCON) em $5 \mathrm{ml}$ de meio de cultura e 10\% SFB. Dois dias após a cultura se tornar totalmente confluente, realizamos uma lesão na camada confluente unicelular com o auxílio de uma lâmina de barbear rígida, pressionada contra o assoalho da placa de cultura, cortando a camada de células e marcando a placa. A lâmina foi então movida cuidadosamente para um lado removendo parte da camada unicelular. As células foram lavadas por duas vezes com PBS para a remoção de material celular deslocado, adicionado meio de cultura experimental (5\% SFB) suplementado de concentrações variáveis de LDLn e LDL-ox. Quatro placas foram utilizadas como controle ou experimentais, para cada ponto do experimento.

Após 24h de incubação, contamos o número de núcleos celulares que cruzaram a linha demarcada na placa, em três campos microscópicos distintos representativos para cada placa e calculamos a média de células que ultrapassaram a linha demarcada.

A purificação da LDLn foi feita a partir do plasma humano utilizando-se o método descrito por Sevanian e cols ${ }^{13}$. A LDLn foi armazenada em temperatura inferior a $-70^{\circ} \mathrm{C}$ até o momento da sua utilização.

As amostras de $\operatorname{LDL}(0,5 \mu \mathrm{g} / \mathrm{ml}$ de proteína) foram submetidas à oxidação com 1,0 $\mu \mathrm{M}$ de 3-morfolinosidnonimina (SIN-1) ou $1,0 \mu \mathrm{M}$ de esperminanonoato (ENO) (SIGMA). As amostras foram incubadas em banho-maria $\left(37^{\circ} \mathrm{C}\right)$ sob agitação constante nos tempos 1, 5, 10, 15, 30, e 60min. A reação foi bloqueada com $100 \mu \mathrm{M}$ de ácido dietileno tetraminopentacético, $100 \mu \mathrm{M}$ de butilhidroxitolueno, 125 unidades/ml de superóxido dismutase e 125 unidades $/ \mathrm{ml}$ de catalase. Todas as amostras foram mantidas em freezer a $-20^{\circ} \mathrm{C}$ até o momento dos experimentos ${ }^{14}$.

Na análise estatística, para a comparação das concentrações foi utilizado o teste não-paramétrico de Kruskal-Wallis, considerado o mais eficiente para amostras independentes e para as comparações múltiplas foi utilizado o teste de Dunn ${ }^{15}$. Foram utilizados testes não-paramétricos porque a suposição de normalidade dos dados foi rejeitada. 0 nível de significância utilizado para os testes foi de $5 \%$.

\section{Resultados}

A figura 1 mostra o efeito das preparações de LDLn sobre a proliferação celular em culturas parcialmente confluentes (60\%) de células endoteliais de artérias coronárias humanas. Após exposição por $72 \mathrm{~h}$ à concentrações variáveis de lipoproteínas, as culturas foram lavadas por 2 vezes com PBS (GIBCO) e coradas pelo método de GIEMSA. A contagem de células foi feita sob microscopia (NIKON-TMS) com aumento de 100 vezes e realizada em três campos microscópicos distintos representativos para cada placa e calculada a média do número de células.

Observamos um efeito inibitório da LDLn sobre a proliferação celular na concentração máxima utilizada, o que pode ser explicado pelo maior tempo de exposição e à oxidação da LDL pelas próprias células endoteliais em cultura.

Nas figuras 2 e 3 mostramos os efeitos da LDL-ox pelo sistema da ENO por 1 e 10min, respectivamente, sobre a proliferação celular, observando em ambos um efeito inibitório proporcional ao tempo de oxidação.

As figuras 4 e 5 mostram os importantes efeitos inibitórios da LDL-ox pelo sistema SIN-1 por tempos de oxidação de 1 e 10min, sobre a proliferação celular.

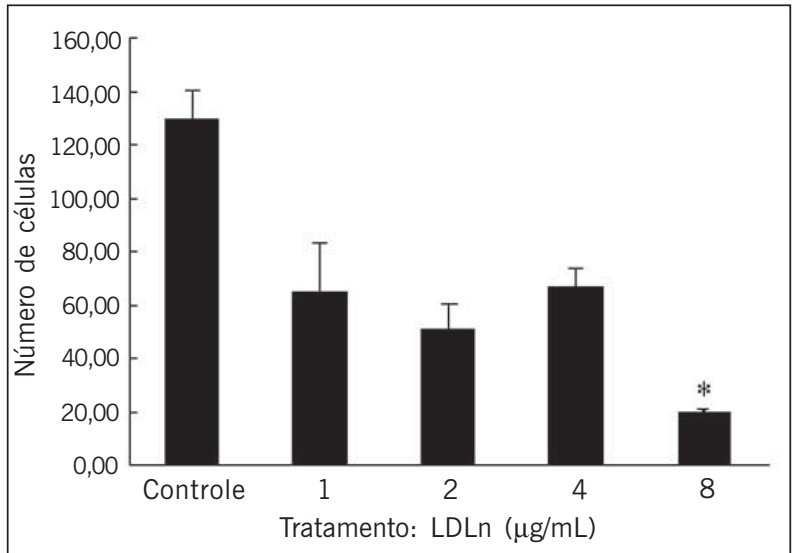

Fig. 1 - Efeito de concentrações variáveis de LDL nativa por 72h sobre número de células por campo microscópico. 0 controle difere do tratamento $8 \mu \mathrm{g} / \mathrm{mL}$ $*(p<0,05)$

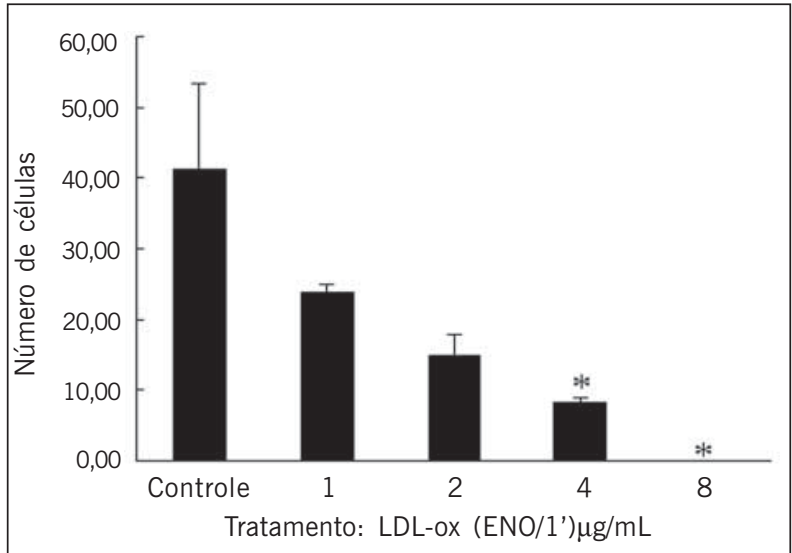

Fig. 2 - Efeito de concentrações variáveis de LDL oxidada (ENO/1') por 72h sobre o número de células por campo microscópico. 0 controle difere a partir dos tratamentos 4 e $8 \mu \mathrm{g} / \mathrm{mL} *(p<0,05)$. 


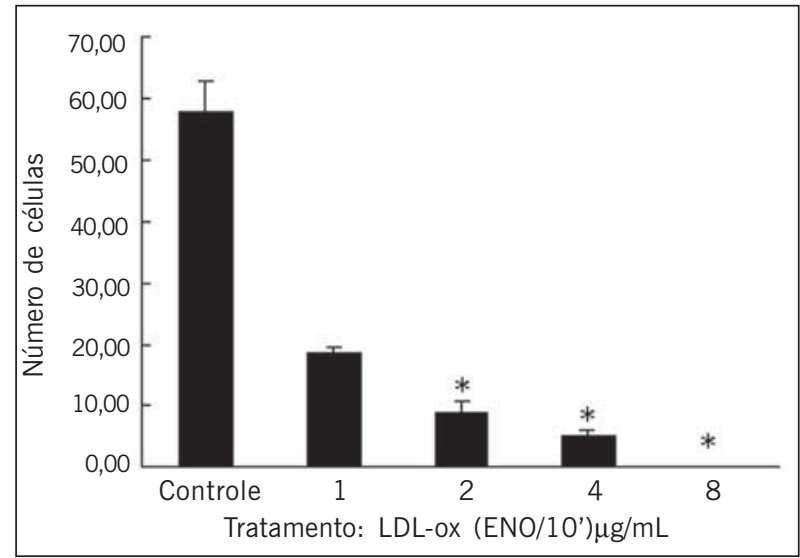

Fig. 3 - Efeito de concentrações variáveis de LDL oxidada (ENO/10') por 72h sobre número de células por campo microscópico. 0 controle difere a partir dos tratamentos 2, 4 e $8 \mu \mathrm{g} / \mathrm{mL}^{*}(\mathrm{p}<0,05)$.

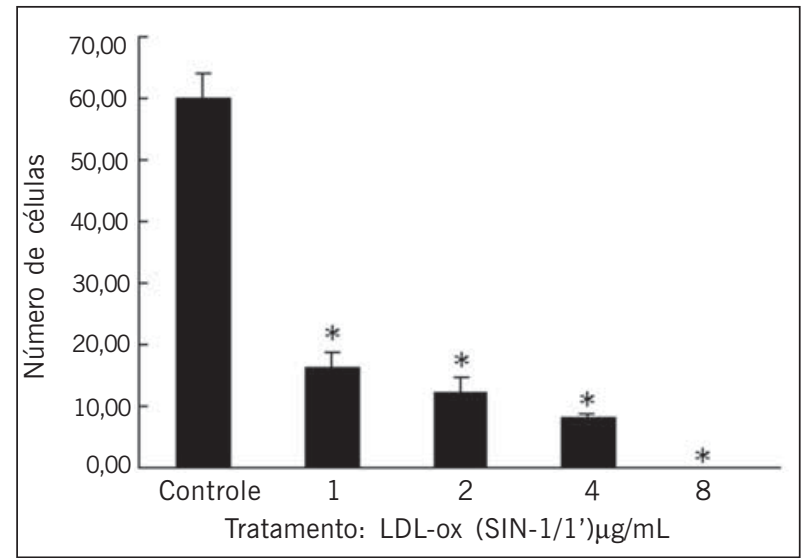

Fig. 4. Efeito de concentrações variáveis de LDL oxidada (SIN-1/1') por $72 \mathrm{~h}$ sobre o número de células por campo microscópico. 0 controle difere dos tratamentos para 1,2 , 4 e $8 \mu \mathrm{g} / \mathrm{mL}^{*}(\mathrm{p}<0,05)$.

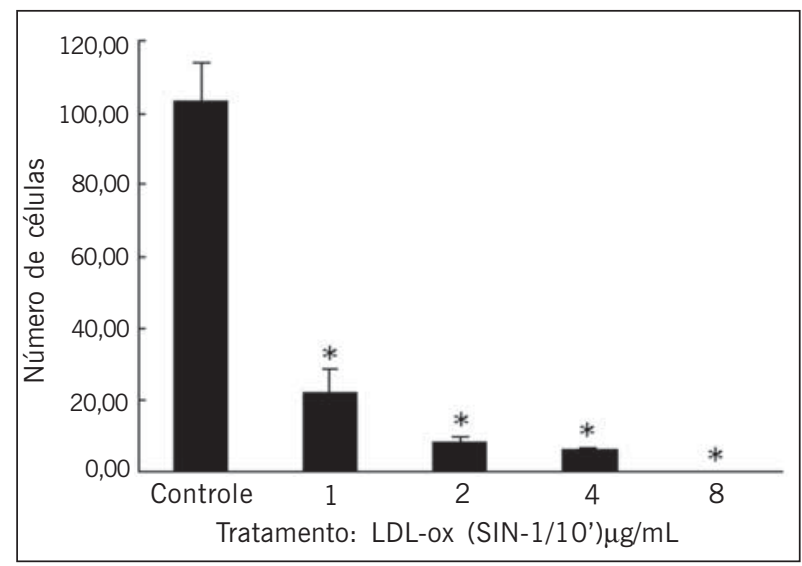

Fig. 5 - Efeito de concentrações variáveis de preparações de LDL oxidada (SIN-1/10') por $72 \mathrm{~h}$ sobre o número de células por campo microscópico. 0 controle difere dos tratamentos para 1,2 , 4 e $8 \mu \mathrm{g} / \mathrm{mL} *(p<0,05)$.

Em resumo, os resultados do tratamento de culturas endoteliais parcialmente confluentes, por 72h, com LDLn e LDL-ox pelos sistemas químicos da ENO e SIN-1 sobre a proliferação celular, mostraram que: a LDL oxidada pela ENO e pela SIN-1 exerceram efeito inibitório na proliferação celular proporcional ao tempo de oxidação e à concentração de LDL-ox; a LDL oxidada pela SIN-1 a LDLn apresentou efeito inibitório somente na maior concentração estudada.

A figura 6 mostra o efeito das preparações de LDLn sobre a motilidade espontânea de células endoteliais de artérias coronárias humanas pelo ensaio de migração celular realizado pela técnica de Burk. Observamos que a LDLn não apresentou nenhum efeito inibitório sobre a migração celular.

As figuras 7 e 8 mostram os efeitos da LDL oxidada pela SIN1 para os tempos de oxidação de 1 e 10min, onde observamos efeitos diretamente relacionados à concentração e ao tempo de oxidação da LDL.

A figura 9 mostra microfotografia representativa do experimento de motilidade celular. Observa-se nitidamente um número muito menor de núcleos celulares que atravessaram a linha demarcada no campo tratado $(B)$ em comparação ao controle $(A)$, demonstrando a migração celular inibida.

Em resumo, os resultados do tratamento de culturas endoteliais com LDLn e LDL-ox pelo sistema SIN-1, por 24 horas, na avaliação da motilidade celular, pela técnica de Burk, mostraram que: a LDLn não apresentou efeito inibitório na migração celular; a LDL-ox pela SIN-1 inibiu a migração celular; e o efeito observado foi proporcional ao tempo de oxidação e à concentração de LDL- ox.

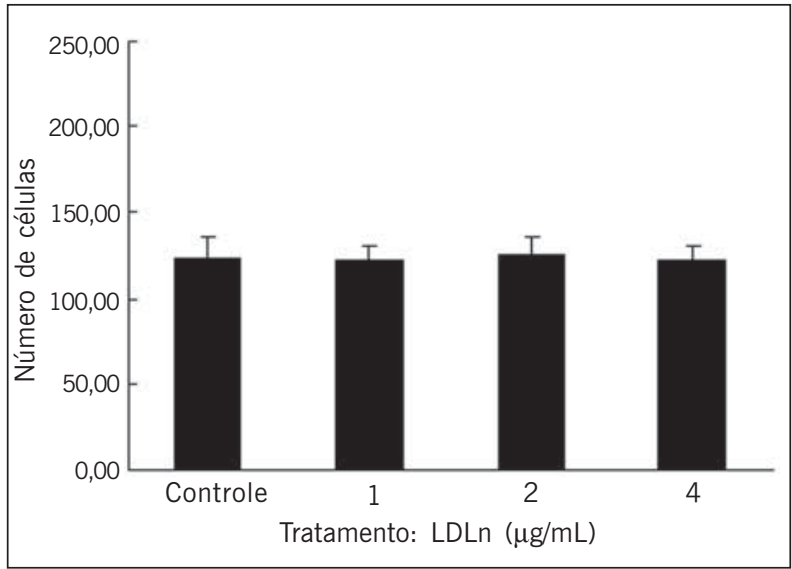

Fig. 6 - Efeito de concentrações variáveis de LDL nativa sobre o número de células que migraram além da linha demarcada. Não há diferença significativa entre os tratamentos $(p=0,8920)$.

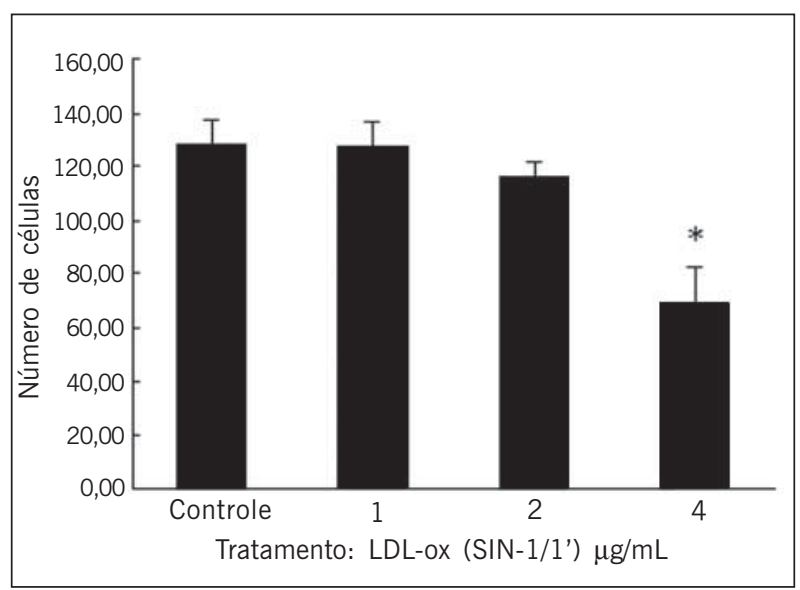

Fig. 7 - Efeito de concentrações variáveis de LDL oxidada (SIN-1/1') sobre o número de células que migraram além da linha demarcada. 0 tratamento $4 \mu \mathrm{g} / \mathrm{mL}$ difere das demais concentrações e do controle* $(p<0,05)$. 


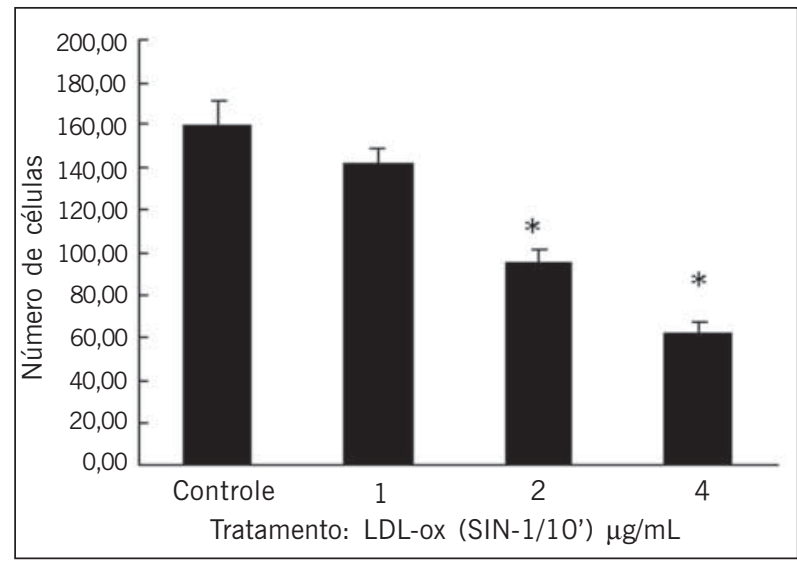

Fig. 8 - Efeito de concentrações variáveis de LDL oxidada (SIN-1/10') sobre o número de células que migraram além da linha demarcada. 0 controle difere dos tratamentos 2 e $4 \mu \mathrm{g} / \mathrm{mL} *(p<0,05)$.

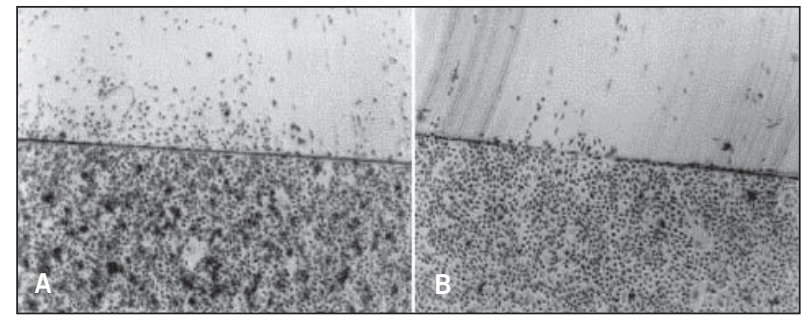

Fig. 9 - Microfotografia de campos celulares representativos da migração celular. A: controle; B: tratamento LDL-ox (SIN-1/ 10'); (aumento 40X).

\section{Discussão}

Recentemente, estudos têm demonstrado que os produtos de oxidação do colesterol, gerados pela modificação oxidativa da LDL, os hidroperóxidos de colesterol, conferem citotoxicidade à partícula e são relevantes para a patogênese e progressão da aterosclerose tendo sido identificados em placas de ateroma e em plasma humano ${ }^{16,17}$.

Os mecanismos moleculares que iniciam a oxidação da LDL in vivo ainda são desconhecidos, no entanto, sabemos que algumas substâncias, como o peroxinitrito, participam efetivamente do processo de modificação da LDL, a peroxidação lipídica ${ }^{18}$. Utilizamos o método de oxidação química da LDL por dois sistemas, SIN-1 e ENO. A SIN-1 é reconhecida como geradora de peroxinitrito e sua utilização nos experimentos pode ser justificada porque esse processo ocorre em fluxo lento e de forma constante, o que seria mais semelhante à via de formação do peroxinitrito em situações fisiológicas. A SIN-1 tem alto grau de oxidação, pois oxida tanto a porção protéica quanto a lipídica da $\operatorname{LDL}^{19,20}$. A ENO, geradora de óxido nítrico, atua promovendo oxidação apenas do conteúdo lipídico da LDL, com baixo grau de oxidação. Assim, a geração de produtos de oxidação pelos dois sistemas produz uma LDL com diferentes graus de oxidação e em diferentes sítios da molécula ${ }^{14}$.

As concentrações de LDL-ox comumente utilizadas em experimentos (25 a $100 \mu \mathrm{g} / \mathrm{mL}$ ) são consideravelmente mais elevadas do que os níveis de LDL-ox encontrados no plasma humano $(0,1 \mu \mathrm{g} / \mathrm{mL})^{21,22}$. Devemos considerar, porém, que nas lesões ateroscleróticas a LDL-ox está localizada e concentrada no espaço subendotelial, porque é retida na matriz extracelular durante a formação da placa de ateroma. Assim, grandes quantidades de
LDL-ox, em concentrações muito maiores que as plasmáticas, estão acumuladas nessa região. A LDL retida nesse microambiente, ideal para oxidação, é mais intensamente oxidada apresentando características similares às LDL oxidadas pelos diversos sistemas utilizados nos estudos experimentais ${ }^{23}$.

Neste estudo, optamos por utilizar baixas concentrações (1 a $8 \mu \mathrm{g} / \mathrm{mL}$ ) de LDL procurando agregar maior relevância aos nossos resultados em função das concentrações estudadas encontraremse mais próximas da situação fisiológica real e assim, investigarmos os seus efeitos biológicos sobre as culturas celulares.

Dados de experimentação obtidos com células endoteliais provenientes de um determinado órgão, não devem ser extrapolados automaticamente para outros sistemas, em decorrência da heterogeneidade dessa linhagem celular. Parte considerável das informações existentes na literatura sobre os complexos mecanismos envolvidos na aterosclerose e na angiogênese humana foi obtida com células endoteliais de cordão umbilical, o que mereceria, portanto, uma certa cautela na interpretação dos resultados: o endotélio do cordão umbilical convive com altíssimas concentrações de hormônios esteróides e baixo regime pressórico, diferentemente do leito endotelial que participa do processo aterosclerótico coronariano ${ }^{11}$. Por esse motivo, optamos pela utilização em nosso trabalho da linhagem de células endoteliais de artérias coronárias humanas.

Culturas de células endoteliais totalmente confluentes podem servir de modelo para estudos in vitro do endotélio vascular, já que as propriedades básicas da célula endotelial são preservadas. As células em cultura parcialmente confluente (60\%) estão em proliferação e nessa fase são muito mais sensíveis a agentes agressores do que no estado de plena integridade da confluência total.

Em nossos experimentos, observamos que LDL-ox inibiu a proliferação e a motilidade in vitro de células endoteliais de artérias coronárias humanas, efeitos estes, diretamente relacionados à concentração e ao grau de oxidação da lipoproteína. Nossos resultados podem sugerir a ocorrência de efeitos similares das LDL-ox presentes nas paredes vasculares, nas placas ateroscleróticas e mesmo circulantes no plasma, reforçando dados da literatura que indicam a participação da LDL-ox em eventos cardiovasculares ${ }^{24,25}$.

Recentemente, foi demonstrado pela primeira vez que níveis plasmáticos elevados de LDL-ox se relacionam diretamente com a instabilidade da placa em lesões ateroscleróticas de artérias coronárias humanas. Os níveis de LDL-ox foram dosados em pacientes com infarto agudo do miocárdio, angina instável, angina estável e controles, revelando uma correlação positiva com a gravidade da síndrome coronariana aguda. Os níveis séricos de LDL-ox eram quatro vezes mais elevados nos pacientes com infarto agudo do miocárdio quando comparados aos controles, sugerindo que LDL-ox circulante pode se constituir em um marcador de gravidade em eventos cardiovasculares ${ }^{26}$.

Considerando os nossos resultados, com a demonstração de que LDL-ox, em concentrações semelhantes às encontradas na fase aguda de síndromes coronarianas, inibe a proliferação e a motilidade de células endoteliais de artérias coronárias humanas in vitro, podemos sugerir que níveis elevados de LDL-ox, por sua citotoxidade, possam interferir negativamente não somente na instabilização da placa aterosclerótica bem como no restabelecimento da integridade vascular pós-injúria, piorando o prognóstico dos pacientes. 
Concluímos, então, que os efeitos da LDL-ox sobre o endotélio coronariano em cultura, inibindo a proliferação e a motilidade celular, mecanismos capitais na reendotelização de áreas lesadas da parede e no processo de angiogênese vascular, são proporcionais à concentração e ao grau de oxidação da LDL e poderíamos ainda, inferir que intervenções clínicas que controlem e preservem a integridade dessas variáveis devam ser perseguidas para minimizar os efeitos deletérios e mudar a história natural da doença arterial coronariana.

A interpretação de nossos dados, porém deve ser feita à luz das limitações inerentes da experimentação in vitro que é muito valiosa no esclarecimento de mecanismos fisiopatológicos, porém não devem ser diretamente extrapolados para a situação in vivo onde atuam um sem número de outros fatores não controlados.

\section{Referências}

1. Ross R, Glonset JA. The pathogenesis of atherosclerosis. N Engl J Med 1976; 295:369-377.

2. Henriksen T, Evensen SA, Carlander B. Injury to human endothelial cells in culture induced by low density lipoproteins. Scand J Clin Lab Invest 1979; 39:361-8.

3. Henriksen T, Evensen SA, Carlander B. Injury to cultured endothelial cells in culture induced by LDL: protection by HDL. Scand J Clin Lab Invest 1979; 39:369-75.

4. Hessler JR, Robertson AL, Chisolm GM. LDL-induced cytotoxicity and its inhibition in human vascular smooth muscle and endothelial cells in culture. Atherosclerosis 1979; 32:213-29.

5. Morel DW, Dicorleto PE, Chisolm GM. Endothelial and smooth muscle cells alter low density lipoprotein in vitro by free radical oxidation. Arteriosclerosis 1984; 4:357-64.

6. Steinbrecher UP, Parthasarathy S, Leake DS et al. Modification of LDL by endothelial cells involves lipid peroxidation and degradation LDL phospholipids. Proc Natl Acad Sci USA 1984; 81:3883-7.

7. Murugesan G, Chisolm GM, Fox PL. Oxidized low density lipoprotein inhibits the migration of aortic endothelial cells in vitro. J Cell Biol 1993; 120:1011-19.

8. Dulak J, Jozkowicz A, Dichtl W et al. VEGF synthesis in vascular smooth muscle cells is enhanced by 7-ketocholesterol and lysophospha tridylcholine independently of their effect on nitric oxide generation. Cardiovasc Res 2002; 5(3):487-8.

9. Dulak J, Jozkowicz A. Regulation of vascular endothelial growth factors synthesis by nitric oxide: facts and controversies. Antiox Redox Signal 2003; 5(1):123-32.

10. Galle J, Heinloth A, Wanner $C$ et al. Dual effect of oxidized LDL on cell cycle in human endothelial cells trough oxidative stress. Kidney Int Suppl 2001; 78:s120-3.

11. Ribatti D, Nico B, Vacca A et al. Endothelial cell heterogeneity and specificity. J of Hematotherapy \& Stem Cell Research 2002; 11:81-90

12. Burk RR. A factor from a transformed cell line that affects cell migration. Proc Natl Acad Sci USA 1973; 70:368-372.

13. Sevanian A, Bittolo-Bon G, Cazollato G et al. LDL is a lipid hydroperoxide-enriched circulating lipoprotein. J Lipid Res 1997; 38:419-28.

14. Oliveira JMA. Efeito da suplementação com vitamina E sobre os níveis de LDL eletronegativa (LDL-), de auto-anticorpos anti-LDL oxidada e marcadores da modificação oxidativa de lipídios e proteínas em pacientes ateroscleróticos. São Paulo,
2002. xxxp. Dissertação (Mestrado) - Faculdade de Ciências Farmacêuticas, Universidade de São Paulo.

15. Rosner B. Fundamentals of Biostatistics - Boston, PWS Publishers, Second edition, 1986.

16. Crisby $\mathrm{M}$, Kallin $\mathrm{B}$, Thyberg $\mathrm{J}$ et al. Cell death in human atherosclerotic plaques involves both oncosis and apoptosis. Atherosclerosis 1997; 130(1-2):17-27.

17. Yasunobu Y, Hayashi K, Shingu T et al. Coronary atherosclerosis and oxidative stress as reflected by autoantibodies against oxidized low-density lipoprotein and oxysterols. Atherosclerosis 2001; 155(2):445-53.

18. Eiserich JP, Patel RP, O'Donnell VB. Pathophysiology of nitric oxide and related species: free radical reactions and modification of biomolecule. Molec Aspect Med 1998; 19:221-357.

19. Feelisch M, Ostrowski J, Noack E. On the mechanism of NO release from sydnonimine. J Cardiovasc Pharmacol 1989; 14(suppl. 11):13-32.

20. Thomas S, Davies MJ, Stocker R. Oxidation and antioxidation of human lowdensity lipoprotein and plasma exposed to 3-morpholinoydnonimine and reagent peroxynitrite. Chem Res Toxicol 1998; 11:484-94.

21. Itabe $\mathrm{H}$, Yamamoto H, Imanaka T et al. Sensitive detection of oxidatively modified low density lipoprotein using a monoclonal antibody. J Lipid Res 1996; 37:45-53.

22. Holvoet $\mathrm{P}$, Donck J, Landeloos $\mathrm{M}$ et al. Correlation between oxidized low density lipoproteins and von Willebrand factor in chronic renal failure. Thromb Haemost 1996; 76:663-9.

23. Yla-Herttuala S, Palinski W, Rosenfeld ME et al. Evidence for the presence of oxidatively modified low density lipoprotein in atherosclerotic lesions of rabbit and man. J Clin Invest 1989; 84:1086-95.

24. Chen $\mathrm{CH}$, Henry PD. Atherosclerosis as a microvascular disease: impaired angiogenesis mediated by suppressed basic fibroblast growth factor expression. Proc Assoc Am Physicians 1997; 109:351-61.

25. Bucal M, Nguy J, Barrios R et al. Impaired adaptative vascular growth in hypercholesterolemic rabbit. Atherosclerosis 1998; 139:243-51.

26. Ehara S, Ueda M, Naruko T et al. Elevated levels of oxidized low density lipoprotein show a positive relationship with the severity acute coronary syndromes. Circulation 2001; 103:1955. 